\title{
Hyperautofluorescent ring in eyes with macular holes
}

This article was published in the following Dove Press journal:

Clinical Ophthalmology

8 August 2013

Number of times this article has been viewed

\section{Tatsuhiko Sato \\ Kazuyuki Emi \\ Yui Osawa \\ Hajime Bando}

Osaka Rosai Hospital Clinical Research Center for Occupational Sensory Organ Disability, Sakai, Japan
Correspondence: Tatsuhiko Sato

Osaka Rosai Hospital Clinical Research

Center for Occupational Sensory Organ

Disability, I I79-3 Nagasone-cho, Kita-ku,

Sakai, 59I-8025, Japan

$\mathrm{Tel}+8172252356 \mathrm{I}$

Fax +8I7 22588720

Email t-satou@orh.go.jp
Background: Fundus autofluorescence studies in eyes with macular holes (MHs) have shown a hyperautofluorescent spot corresponding to the hole and a hypoautofluorescent ring corresponding to the fluid cuff surrounding the hole. The purpose of this report is to present three cases of $\mathrm{MH}$ with a different fundus autofluorescence pattern.

Case reports: Case 1 was a 62-year-old woman who did not know the duration of the $\mathrm{MH}$ in her left eye. Her decimal best-corrected visual acuity (BCVA) was 0.08 . The left eye had a one-half disc diameter $\mathrm{MH}$ with a depigmented ring surrounding the area of the fluid cuff. Fundus autofluorescence showed a hyperautofluorescent ring corresponding to the depigmented ring. After vitrectomy, fundus autofluorescence showed the same size hyperautofluorescent ring despite the decreased size of the opened MH. Case 2 was a 69-year-old woman who had been diagnosed with $\mathrm{MH}$ in the right eye 13 years earlier. Her decimal BCVA was 0.1 . The right eye had a one-half disc diameter $\mathrm{MH}$ with a depigmented ring surrounding the area of the fluid cuff. Fundus autofluorescence showed a hyperautofluorescent ring corresponding to the depigmented ring. Postoperative fundus autofluorescence showed the same size hyperautofluorescent ring despite the hole being closed. The decimal BCVA was 0.2 in her right eye 6 months after vitrectomy. Case 3 was a 71-year-old woman who had been diagnosed with $\mathrm{MH}$ in the right eye 15 years earlier. Her decimal BCVA was 0.1 . The right eye had a one-half disc diameter $\mathrm{MH}$ with a depigmented ring which corresponded with a hyperautofluorescent ring. Postoperative fundus autofluorescence showed the same size hyperautofluorescent ring despite the hole being closed. The decimal BCVA was 0.2 in her right eye 6 months after vitrectomy.

Conclusion: Our findings suggest that a hyperautofluorescent ring in eyes with MHs may be an indicator of a poor surgical prognosis both anatomically and functionally.

Keywords: macular hole, fundus autofluorescence, vitrectomy, predictor

\section{Introduction}

Macular hole $(\mathrm{MH})$ is a full-thickness defect of the foveal retina that causes a severe reduction in central vision. The prevalence of $\mathrm{MH}$ has been reported to be $0.09 \%-0.17 \% .^{1-4}$ The established treatment for $\mathrm{MH}$ is pars plana vitrectomy combined with internal limiting membrane peeling followed by intravitreal gas tamponade. Recent studies have shown that the anatomic success rate after primary pars plana vitrectomy is $89 \%-97 \% .^{5-9}$ The size and duration of the $\mathrm{MH}$ are preoperative factors that are significantly associated with postoperative anatomic $\mathrm{MH}$ closure. ${ }^{10-13}$ The size of MH can be estimated by biomicroscopy or more quantitatively by optical coherence tomography. However, the duration of $\mathrm{MH}$ is more difficult to determine accurately because some patients are unaware of the reduced vision until the fellow eye is accidentally covered. 
Fundus autofluorescence is a relatively new noninvasive method of assessing the retina and retinal pigment epithelium and has been used to evaluate eyes with $\mathrm{MH} .{ }^{14-17}$ Fundus autofluorescence is believed to be an indicator of the level of lipofuscin in retinal pigment epithelium cells, ${ }^{18,19}$ and earlier studies on eyes with MH reported a hyperautofluoresence corresponding to the full-thickness $\mathrm{MH}$ and a hypoautofluoresence corresponding to the surrounding fluid cuff. ${ }^{14-17}$

We report a new fundus autofluorescence pattern in three eyes with $\mathrm{MH}$, namely a hyperautofluorescent ring. We suggest that this fundus autofluorescence pattern may be an indicator of a poor postoperative prognosis for both anatomic and functional recovery of the retina.

\section{Case reports}

The procedures used in this study conformed to the tenets of the Declaration of Helsinki, and were approved by the institutional review board of Osaka Rosai Hospital. The three patients were informed on the nature and possible consequences of the procedures to be used. A signed informed consent was obtained from all patients.

\section{Case I}

A 62-year-old woman was referred to our department with a diagnosis of MH in her left eye. She had not noticed the decrease in her central vision until it was pointed out by an optometrist. At presentation, her decimal best-corrected visual acuity (BCVA) was 1.0 in the right eye and 0.08 in the left eye. Fundus photographs and fundus autofluorescence images were taken with the Optos ${ }^{\circledR} 200 \mathrm{Tx}$ (Optos, Scotland, United Kingdom), and optical coherence tomography was performed with the Cirrus ${ }^{\mathrm{TM}}$ HD OCT (Carl Zeiss Meditec Japan, Tokyo, Japan). The left eye had a large MH with an operculum (Figure 1A and E). The minimal and maximal diameters of the $\mathrm{MH}$ were $864 \mu \mathrm{m}$ and $1,345 \mu \mathrm{m}$, respectively, and a depigmented ring was observed surrounding the area of the fluid cuff (Figure 1A and E). Fundus autofluorescence showed a hyperautofluorescent ring corresponding to the depigmented ring (Figure 1A and C).

Combined phacoemulsification and vitrectomy with a 25 -gauge system was performed on the left eye under local anesthesia. Core vitrectomy following the creation of posterior vitreous detachment was performed with an ultrahighspeed cutter (5,000 cycles per minute, Alcon Laboratories Inc, Fort Worth, TX, USA). The internal limiting membrane was stained with $0.025 \%$ Brilliant Blue $\mathrm{G}^{20}$ and peeled within the vascular arcade. The peripheral retina was examined for 360 degrees with scleral indentation to search for any

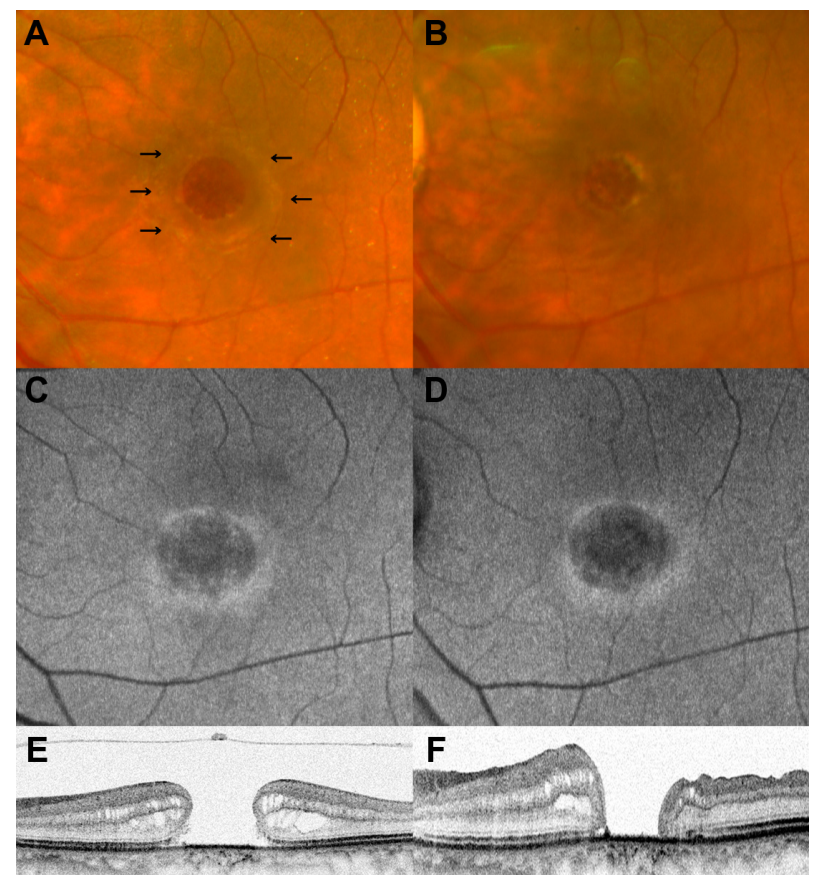

Figure I Fundus findings of the left eye in case I before (A, C, and $\mathbf{E})$ and 6 months after (B, D, and $\mathbf{F}$ ) vitrectomy. ( $\mathbf{A}$ and $\mathbf{B})$ Fundus photographs. The preoperative fundus photograph shows the depigmented ring surrounding the area of the fluid cuff (black arrows). (C and D) FAF images. The preoperative FAF image shows the hyperautofluorescent ring corresponding to the depigmented ring. The postoperative FAF shows the same size of hyperautofluorescent ring as the preoperative one. (E and F) Optical coherence tomographic images. An anatomical closure of the $\mathrm{MH}$ was not achieved after the vitrectomy although the $\mathrm{MH}$ size became smaller. Abbreviations: $\mathrm{FAF}$, fundus autofluorescence; $\mathrm{MH}$, macular hole.

retinal breaks. The vitreous cavity was filled with $20 \%$ sulfur hexafluoride, and the patient was instructed to maintain a prone position for as long as possible.

An anatomic closure of the $\mathrm{MH}$ was not achieved. She did not want any additional treatment, and the $\mathrm{MH}$ remained open for 6 months after the vitrectomy (Figure 1B and F). Fundus autofluorescence at this time showed the same size hyperautofluorescent ring as the preoperative one in spite of a decrease in the postoperative size of the opened $\mathrm{MH}$ (Figure 1D). The decimal BCVA of the left eye 6 months after the vitrectomy was poor at 0.08 .

\section{Case 2}

A 69-year-old woman was referred to our department with a diagnosis of $\mathrm{MH}$ in her right eye. She reported that her central vision had decreased 13 years earlier, and her doctor informed her that no treatment was available to close the $\mathrm{MH}$ at that time. At our initial examination, her decimal BCVA was 0.1 in the right eye and 1.0 in the left eye. The minimal and maximal diameter of the MH were $681 \mu \mathrm{m}$ and $1,555 \mu \mathrm{m}$, respectively (Figure 2A and E). Optical coherence tomography showed disruptions of the photoreceptor inner 


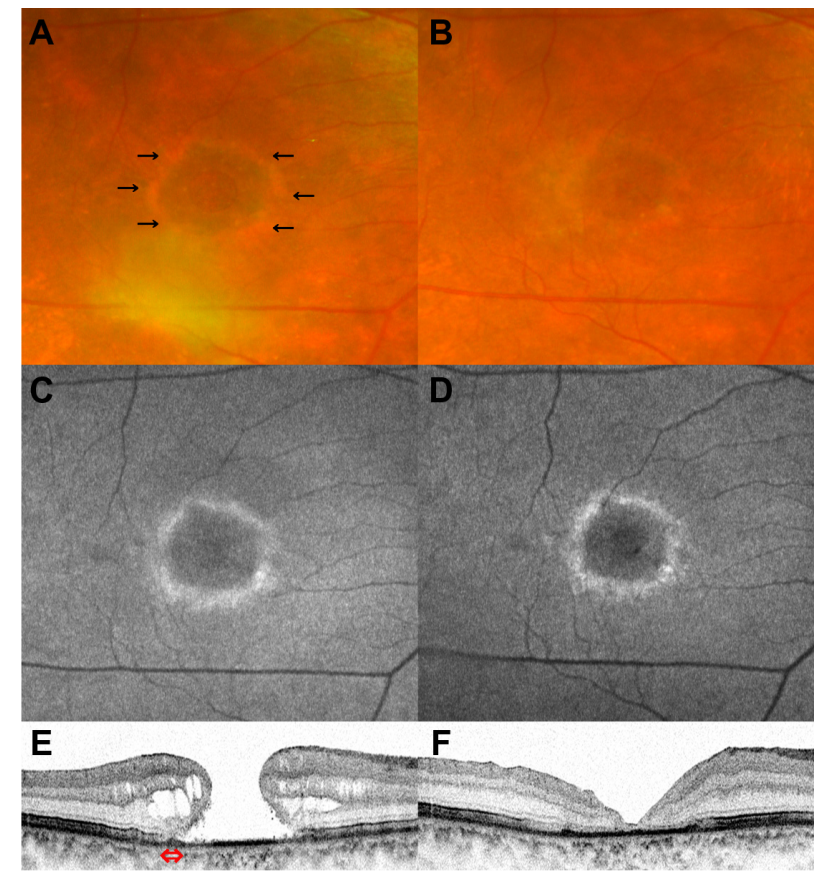

Figure 2 Fundus findings of the right eye in case 2 before (A, C, and $\mathbf{E})$ and 6 months after (B, D, and F) vitrectomy. (A and B) Fundus photographs. The preoperative fundus photograph shows the depigmented ring surrounding the area of fluid cuff (black arrows). (C and D) FAF images. The preoperative FAF image shows the hyperautofluorescent ring corresponding to the depigmented ring. The postoperative FAF shows the same size of hyperautofluorescent ring as the preoperative one. (E and $\mathbf{F}$ ) OCT images. The preoperative OCT image shows the disruptions of the photoreceptor inner and outer segment junction line and the external limiting membrane line at the edge of the cuff of subretinal fluid (red double-headed arrow). The MH was anatomically closed but the OCT images do not show a recovery of the photoreceptor inner and outer segment junction line and the external limiting membrane line 6 months after the vitrectomy.

Abbreviations: $\mathrm{FAF}$, fundus autofluorescence; $\mathrm{MH}$, macular hole; OCT, optical coherence tomography.

and outer segment junction (IS/OS) line and the external limiting membrane line at the edge of the cuff of subretinal fluid (Figure 2E). A depigmented ring was seen surrounding the area of the fluid cuff, and fundus autofluorescence showed a hyperautofluorescent ring corresponding to the depigmented ring (Figure 2A and $\mathrm{C}$ ).

Combined phacoemulsification and vitrectomy was performed with a 25 -gauge ultrahigh-speed cutter on the right eye under local anesthesia. After core vitrectomy followed by the creation of posterior vitreous detachment, peripheral vitrectomy and 360 degree vitreous shaving with scleral indentation was performed. The internal limiting membrane within the vascular arcade was peeled assisted by Brilliant Blue $\mathrm{G}$ staining. The vitreous cavity was filled with $12 \%$ perfluoropropane. She was instructed to maintain a prone position for as long as possible.

The MH was anatomically closed but the optical coherence tomography images did not show any recovery of the IS/OS line or the external limiting membrane line 6 months after vitrectomy (Figure 2B and F). Fundus autofluorescence showed the same size of hyperautofluorescent ring as seen preoperatively despite the $\mathrm{MH}$ being closed (Figure 2D). The decimal BCVA of the right eye 6 months after the vitrectomy was 0.2 .

\section{Case 3}

A 71-year-old woman was referred to our department with a diagnosis of $\mathrm{MH}$ in her right eye. She reported that her central vision had decreased 15 years earlier, and her doctor informed her that no treatment was available to close the $\mathrm{MH}$ at that time. At our initial examination, her decimal BCVA was 0.1 in the right eye and 0.8 in the left eye. The minimal and maximal diameter of the MH was $818 \mu \mathrm{m}$ and 1,494 $\mu \mathrm{m}$, respectively (Figure 3A and E). Optical coherence tomography showed disruptions of the IS/OS line and the external limiting membrane line at the edge of the cuff of subretinal fluid (Figure 3E). A depigmented ring and corresponding hyperautofluorescent ring were observed (Figure 3A and C).

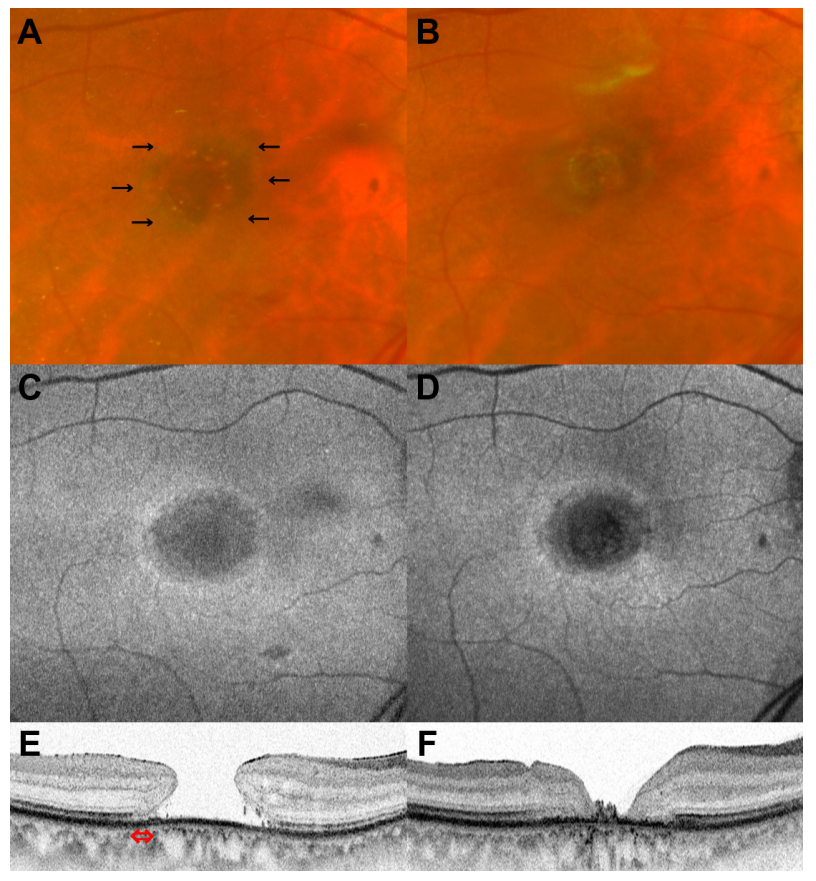

Figure 3 Fundus findings of the right eye in case 3 before $(\mathbf{A}, \mathbf{C}$, and $\mathbf{E})$ and 6 months after (B, D, and F) vitrectomy. (A and B) Fundus photographs. The preoperative fundus photograph shows the depigmented ring surrounding the area of fluid cuff (black arrows). (C and D) FAF images. The preoperative FAF image shows the hyperautofluorescent ring corresponding to the depigmented ring. The postoperative FAF shows the same size of hyperautofluorescent ring as the preoperative one. (E and F) OCT images. The preoperative OCT images show disruptions of the photoreceptor inner and outer segment junction line and the external limiting membrane line at the edge of the cuff of subretinal fluid (red double-headed arrow). The MH was anatomically closed, and the OCT image does not show a recovery of the photoreceptor inner and outer segment junction line and the external limiting membrane line 6 months after the vitrectomy.

Abbreviations: FAF, fundus autofluorescence; $\mathrm{MH}$, macular hole; OCT, optical coherence tomography. 
Combined phacoemulsification and vitrectomy was performed on the right eye with a 25 -gauge ultrahigh-speed cutter under local anesthesia. After core vitrectomy followed by the creation of posterior vitreous detachment, peripheral vitrectomy and 360 degree vitreous shaving was performed. The inverted internal limiting membrane flap technique ${ }^{12}$ assisted by Brilliant Blue G staining was performed. The vitreous cavity was filled with $20 \%$ sulfur hexafluoride, and she was instructed to maintain a prone position for as long as possible.

Postoperatively, the MH was anatomically closed but a recovery of both the IS/OS line and the external limiting membrane line was not seen 6 months after vitrectomy (Figure 3B and F). Fundus autofluorescence showed the same size of hyperautofluorescent ring as seen preoperatively despite the MH being closed (Figure 3D). The decimal BCVA of the right eye 6 months after the vitrectomy was 0.2 .

\section{Discussion}

As best we know, a hyperautofluorescent ring in eyes with MH has not been reported, and the surgical outcomes of treating such eyes have also not been reported. Our findings showed that the MHs in two of the three cases were anatomically closed although the visual acuities were still poor. The postoperative fundus autofluorescence images were unchanged, with the same size hyperautofluorescent ring in spite of a decrease in MH size in one eye and successful closure of the $\mathrm{MH}$ in two eyes.

The intensity of the fundus autofluorescence image is believed to be an indicator of the lipofuscin level within the retinal pigment epithelium cells. ${ }^{18,19}$ It is generally accepted that lipofuscin is the byproduct of degradation of the photoreceptor outer segments phagocytosed by the retinal pigment epithelium cells. ${ }^{21}$ Thus, an increase in intensity of the fundus autofluorescence is interpreted as higher turnover of photoreceptor outer segments or impaired metabolic activity of the retinal pigment epithelium cells. ${ }^{16}$ In addition, the intensity of the fundus autofluorescence image is increased when retinal tissues are absent because the luteal pigment and the neurosensory retinal tissue attenuate the fundus autofluorescence signal. ${ }^{16}$ On the other hand, a decrease in the intensity or absence of fundus autofluorescence signal is interpreted to result from lack of photoreceptors, loss of metabolic ability of the retinal pigment epithelium cells, or blocking of the fundus autofluorescence signal from the retinal pigment epithelium. ${ }^{16}$

In earlier studies, it was demonstrated that the intensity of fundus autofluorescence in eyes with $\mathrm{MH}$ is increased corresponding to the hole because of loss of the foveal tissues and is decreased in the surrounding fluid cuff due to the presence of subretinal fluid and/or thickening of the neurosensory retina. ${ }^{14-17}$ On the other hand, the preoperative fundus autofluorescence in our three cases with MH did not have an increase in intensity over the hole but a hyperautofluorescent ring surrounding the area of the fluid cuff.

The reason for the difference in fundus autofluorescence pattern between the earlier reports and our three cases was not determined. However, it is possible that the difference is because our cases were long-standing MHs; cases 2 and 3 were diagnosed with MH 13 and 15 years earlier, respectively, and case 1 had a large $\mathrm{MH}$ at presentation although the patient did not know the exact duration of the MH. Earlier studies on the natural course of untreated MHs showed that MHs detected early were generally small at $<400 \mu \mathrm{m}$, and that they enlarge and develop into mature $\mathrm{MHs}$ of $\geq 400 \mu \mathrm{m} .{ }^{22-24}$ In our cases, the preoperative minimal diameters of our MHs were $864 \mu \mathrm{m}, 681 \mu \mathrm{m}$, and $818 \mu \mathrm{m}$.

Thus, we suggest that photoreceptors were absent in the central area of the hole for a long time in our three cases. In most cases of $\mathrm{MH}$, the central area has a hyperautofluorescent image because of the absence of foveal tissues that attenuate the fundus autofluorescence signal. However, we suggest that the intensity of the central fundus autofluorescence image in our three cases was not increased because of the prolonged absence of photoreceptors. In addition, Chung et al discussed the possibility that the function of bare central retinal pigment epithelium would be compromised from photo-oxidative damage or long-term lack of normal interaction with retinal tissues. $^{25}$

Shiragami et $a{ }^{17}$ reported on the surgical results of 78 cases with $\mathrm{MH}$, and showed a preoperative hyperfluorescence of the fundus autofluorescence corresponding to the central area of the full-thickness MH. The mean duration of the MHs was 3.4 months, and the mean preoperative visual acuity was 0.61 in logarithm of the minimal angle of resolution ( $\log$ MAR) units. The mean visual acuity was $0.17 \log$ MAR units 6 months after vitrectomy. A continuous external limiting membrane line was seen in $97.4 \%$ of the eyes and a continuous IS/OS line was seen in $83.3 \%$ of the eyes 6 months after the vitrectomy. In two of our cases, the duration of the $\mathrm{MH}$ was over 10 years. The mean preoperative and postoperative visual acuity of the three cases was 1.03 and 0.83 in $\operatorname{logMAR}$ units, respectively. One of the three cases was not closed anatomically, and the other two cases did not show a recovery of both the external limiting membrane and IS/OS lines 6 months after successful vitrectomy. These results 
suggest that MHs with a hyperautofluorescent ring have poor outcomes both anatomically and functionally.

The long-term observational study with a follow-up period of $\geq 5$ years by Casuso et $\mathrm{al}^{24}$ reported that retinal pigment epithelium atrophy developed beneath the rim of the fluid cuff in eyes with untreated $\mathrm{MH}$. They also mentioned that MHs generally enlarge and develop into mature MHs $(\geq 400 \mu \mathrm{m})$, and visual acuity generally stabilizes at the $20 / 200$ to 20/400 level. Our findings are in good agreement with their observations: in the three cases with the hyperautofluorescent ring corresponding to the depigmented ring, the size of the holes was $\geq 400 \mu \mathrm{m}$ and the preoperative decimal BCVA ranged from 0.08 to 0.1 . If the hyperautofluorescent ring results from retinal pigment epithelium atrophy, it is no wonder that the size of the hyperautofluorescent ring remained unchanged after vitrectomy despite the decrease in $\mathrm{MH}$ size or successful closure of the $\mathrm{MH}$.

In conclusion, we reported three $\mathrm{MH}$ cases with a hyperautofluorescent ring. Our findings suggest that this fundus autofluorescence pattern in eyes with $\mathrm{MH}$ may be an indicator of poor surgical prognoses both anatomically and functionally.

\section{Acknowledgment}

This study was supported by Clinical Research Center for Occupational Sensory Organ Disability from the Ministry of Health, Labour and Welfare, Japan.

\section{Disclosure}

The authors have no proprietary interest or conflict of interest in any aspect of this report.

\section{References}

1. la Cour M, Friis J. Macular holes: classification, epidemiology, natural history and treatment. Acta Ophthalmol Scand. 2002;80(6):579-587.

2. Wang S, Xu L, Jonas JB. Prevalence of full-thickness macular holes in urban and rural adult Chinese: the Beijing Eye Study. Am J Ophthalmol. 2006;141(3):589-591.

3. Sen P, Bhargava A, Vijaya L, George R. Prevalence of idiopathic macular hole in adult rural and urban south Indian population. Clin Experiment Ophthalmol. 2008;36(3):257-260.

4. McCannel CA, Ensminger JL, Diehl NN, Hodge DN. Population-based incidence of macular holes. Ophthalmology. 2009;116(7):1366-1369.

5. Merkur AB, Tuli R. Macular hole repair with limited nonsupine positioning. Retina. 2007;27(3):365-369.

6. Madgula IM, Costen M. Functional outcome and patient preferences following combined phaco-vitrectomy for macular hole without prone posturing. Eye (Lond). 2008;22(8):1050-1053.
7. Christensen UC, Kroyer K, Sander B, et al. Value of internal limiting membrane peeling in surgery for idiopathic macular hole stage 2 and 3 : a randomised clinical trial. Br J Ophthalmol. 2009;93(8):1005-1015.

8. Heath G, Rahman R. Combined 23-gauge, sutureless transconjunctival vitrectomy with phacoemulsification without face down posturing for the repair of idiopathic macular holes. Eye (Lond). 2010;24(2):214-220.

9. Kumar A, Gogia V, Shah VM, Nag TC. Comparative evaluation of anatomical and functional outcomes using brilliant blue $\mathrm{G}$ versus triamcinolone assisted internal limiting membrane peeling in macular hole surgery in Indian population. Graefes Arch Clin Exp Ophthalmol. 2011;249(7):987-995.

10. Wendel RT, Patel AC, Kelly NE, Salzano TC, Wells JW, Novack GD. Vitreous surgery for macular holes. Ophthalmology. 1993;100(11): 1671-1676.

11. Ryan EH Jr, Gilbert HD. Results of surgical treatment of recentonset full-thickness idiopathic macular holes. Arch Ophthalmol. 1994;112(12):1545-1553.

12. Michalewska Z, Michalexski J, Adelman R, Nawrocki J. Inverted internal limiting membrane flap technique for large macular holes. Ophthalmology. 2010;117(10):2018-2025.

13. Salter AB, Folgar FA, Weissbrot J, Wald KJ. Macular hole surgery prognostic success rates based on macular hole size. Ophthalmic Surg Lasers Imaging. 2012;43(3):184-189.

14. von Ruckmann A, Fitzke FW, Gregor ZJ. Fundus autofluorescence in patients with macular holes imaged with a laser scanning ophthalmoscope. Br J Ophthalmol. 1998;82(4):346-351.

15. Ciardella AP, Lee GC, Langton K, Sparrow J, Chang S. Autofluorescence as a novel approach to diagnosing macular holes. Am J Ophthalmol. 2004;137(5):956-959.

16. Wakabayashi T, Ikuno Y, Sayanagi K, Soga K, Oshima Y, Tano Y. Fundus autofluorescence related to retinal morphological and functional changes in idiopathic macular holes. Acta Ophthalmol. 2008;86(8): 897-901.

17. Shiragami C, Shiraga F, Nitta E, Fukuda K, Yamaji H. Correlation of increased fundus autofluorescence signals at closed macula with visual prognosis after successful macular hole surgery. Retina. 2012;32(2): $281-288$.

18. Delori FC, Dorey CK, Staurenghi G, Arend O, Goger DG, Weiter JJ. In vivo fluorescence of the ocular fundus exhibits retinal pigment epithelium lipofuscin characteristics. Invest Ophthalmol Vis Sci. 1995;36(3): 718-729.

19. von Ruckmann A, Fitzke FW, Bird AC. Distribution of fundus autofluorescence with a scanning laser ophthalmoscope. Br J Ophthalmol. 1995;79(5):407-412.

20. Enaida H, Hisatomi T, Hata Y, et al. Brilliant blue G selectively stains the internal limiting membrane/brilliant blue G-assisted membrane peeling. Retina. 2006;26(6):631-636.

21. Sparrow JR, WuY, Kim CY, Zhou J. Phospholipid meets all-trans-retinal: the making of RPE bisretinoids. J Lipid Res. 2010;51(2):247-261.

22. Johnson RN, Gass JD. Idiopathic macular holes. Observations, stages of formation, and implications for surgical intervention. Ophthalmology. 1988;95(7):917-924.

23. Gass JD. Reappraisal of biomicroscopic classification of stages of development of a macular hole. Am J Ophthalmol. 1995;119(6):752-759.

24. Casuso LA, Scott IU, Flynn HW Jr, et al. Long-term follow-up of unoperated macular holes. Ophthalmology. 2001;108(6):1150-1155.

25. Chung H, Shin CJ, Kim JG, Yoon YH, Kim HC. Correlation of microperimetry with fundus autofluorescence and spectral-domain optical coherence tomography in repaired macular holes. $\mathrm{Am} \mathrm{J}$ Ophthalmol. 2011;151(1):128-136. 


\section{Publish your work in this journal}

Clinical Ophthalmology is an international, peer-reviewed journal covering all subspecialties within ophthalmology. Key topics include: Optometry; Visual science; Pharmacology and drug therapy in eye diseases; Basic Sciences; Primary and Secondary eye care; Patient Safety and Quality of Care Improvements. This journal is indexed on
PubMed Central and CAS, and is the official journal of The Society of Clinical Ophthalmology (SCO). The manuscript management system is completely online and includes a very quick and fair peer-review system, which is all easy to use. Visit http://www.dovepress.com/ testimonials.php to read real quotes from published authors. 